\title{
Predictive Markers of Missed Miscarriage
}

\author{
Agamurad A. Orazmuradov, $\mathrm{PhD}, \mathrm{ScD}^{1}$; Anastasiya N. Akhmatova, $\mathrm{PhD}^{1}$; Khalid Haddad ${ }^{1}$; \\ Alexander M. Lopatin ${ }^{1}$; Irina V. Bekbaeva ${ }^{1}$; Gayane A. Arakelyan ${ }^{1}$; Marianna Z. Abitova ${ }^{1}$; \\ Sergey I. Kyrtikov ${ }^{1}$; Nozimabonu M. Zokirova ${ }^{1}$; Aleksey A. Lukaev, PhD ${ }^{2}$ \\ 'Peoples' Friendship University of Russia (RUDN University), Moscow, Russia \\ ${ }^{2}$ Mytishchi City Clinical Hospital Mytishchi, Moscow Region, Russia
}

\begin{abstract}
The aim of this study was to find useful the serological markers for missed miscarriage (MM) in order to predict the outcome of pregnancy. The study included 141 pregnant women aged between 18 and 45 years at gestational age under 11 weeks. All women were divided into 3 groups. Group 1 included 68 women with MM; Group 2 included 43 women with spontaneous miscarriage; Group 3 included 30 pregnant women without pathology. Proteomic analysis of the blood serum was performed using liquid chromatography-mass spectrometry. The results of our study show that immunoglobulin kappa variable 3-15 (KV315) can be considered as the most promising serologic marker for MM in early gestation. The potential role of KV315 as the serological marker is very important for predicting the course of pregnancy.(International Journal of Biomedicine. 2021;11(1):65-67.)
\end{abstract}

Key Words: missed miscarriage $\bullet$ serological markers • immunoglobulin kappa variable 3-15

For citation: Orazmuradov AA, Akhmatova AN, Haddad Kh, Lopatin AM, Bekbaeva IV, Arakelyan GA, Abitova MZ, Kyrtikov SI, Zokirova NM, Lukaev AA. Predictive Markers of Missed Miscarriage. International Journal of Biomedicine. 2021;11(1):6567. doi:10.21103/Article11(1)_ShC

\section{Abbreviations}

MM, missed miscarriage; KV315, immunoglobulin kappa variable 3-15; ANA, antinuclear antibodies; LC, light chain; FLC, free light chains: LC-MS, liquid chromatography-mass spectrometry; AMBP, alpha-1-microglobulin/bikunin precursor; TTR, transthyretin; apoA-IV, apolipoprotein A-14; ITIH4, inter-alpha-trypsin inhibitor heavy chain H4.

\section{Introduction}

Missed miscarriage (MM) is one of the unsolved problems both in the Russian Federation and in the world. MM is a specific type of miscarriage and may occur in up to $50 \%$ of all women with miscarriages. There is still a lack of biomarkers with predictive value for asymptomatic patients before this event occurs, but multiple etiologic factors-including genetic risk factors, immunological factors, endocrine and blood disorders, uterine abnormalities, infections, and environmental factors - have been identified for MM.

"Corresponding author: Aleksey A. Lukaev, PhD. Mytishchi municipal clinical hospital, Mytishchi, Moscow Region, Russia E-mail: aleksei_lukaev@mail.ru
It is well known that pregnancy outcome and fetal development largely depend on the state of the mother's immune system. Thus, the more pronounced the changes in the antibody content, the more often adverse pregnancy outcomes are observed. ${ }^{(1,2)}$ In recent years, immunological abnormalities have begun to be considered as etiological factors of miscarriage, in particular, the circulation in the blood of ANA or anti-DNA antibodies. Increased titers of anti-DNA antibodies in various autoimmune and infectious diseases can cause inflammatory changes in the placenta and trigger a reaction of fetal rejection. ${ }^{(3,4)}$

In the modern literature, there are few data on serological markers of MM, which creates the prerequisites for conducting this study aimed at finding the prognostic serological markers for MM in order to predict the outcome of pregnancy. 


\section{Materials and Methods}

In the period from April 2020 to December 2020, a group of 141 pregnant women aged between 18 and 45 years at gestational age under 11 weeks were examined. All women were divided into 3 groups. Group 1 (Gr1) included 68 women with MM; Group 2 (Gr2) included 43 women with spontaneous miscarriage; Group 3 included 30 women without pathology (control group [CG]).

Inclusion criteria were MM, spontaneous miscarriage or physiological pregnancy at gestational age under 11 weeks. Exclusion criteria were autoimmune diseases, infectious diseases, and diseases of the thyroid gland, including thyroid dysfunction.

Examination of patients included clinical methods, questionnaire survey, analysis of case histories, laboratory tests (clinical blood test, biochemical blood test, urine test, and determination of antibody titer to the TORCH complex), and pelvic ultrasound. All women underwent an assessment of vaginal microcenosis and the quantitative and qualitative composition of the biotope of the cervical discharge. Proteomic analysis of the blood serum was performed using LC-MS.

The study was conducted in accordance with ethical principles of the WMA Declaration of Helsinki (1964, ed. 2013) and approved by the RUDN University Ethics Committee. Written informed consent was obtained from all participants.

Statistical analysis was performed using the Statistica 8.0 software package (StatSoft Inc, USA). A value of $P<0.05$ was considered statistically significant.

\section{Results and Discussion}

For the analysis of potentially significant markers, we studied serological markers found in all 3 groups; in particular, we compared the ratios of protein concentrations in the study groups. If the concentration ratio of the investigated marker in the Gr1/CG pair was close to 1 or equal to 1 , then the marker was considered nonspecific; if the concentration ratio of the investigated marker in the Gr1/Gr2 pair was close to 1 or equal to 1 , then the marker was also considered non-specific; however, if the studied ratio was close to 1 or equal to 1 in the Gr1/Gr2 pair and at the same time $>1$ in the Gr1/CG pair, then the marker was considered potentially specific. As can be seen from Table 1, the most specific serorlogical marker of MM may be KV315, since the ratio of its concentration was 4.936 in the Gr1/CG pair, 1.243 in the Gr1/Gr2 pair, and 3.971 in the Gr2/CG pair. For all other markers, differences between groups were less pronounced.

The potential role of KV315 as the serological marker is very important for predicting the course of pregnancy. KV315 (P01624-KV315_HUMAN) (V region of the variable domain of immunoglobulin light chains that participates in the antigen recognition $)^{(5)}$ is involved in a number of potentially interesting pathways such as Initial triggering of complement, classical antibody-mediated complement activation, scavenging heme from plasma, FCGR activation, platelet activation, acute-phase responses, FCERI mediated NF-kB activation degranulation, signaling, aggregation, and the creation of $\mathrm{C} 4$ and $\mathrm{C} 2$ activators, regulation of complement cascade, etc ${ }^{(6)}$

Table 1.

The specific serorlogical marker of MM

\begin{tabular}{|c|c|c|c|c|c|}
\hline Group pair & apoA-IV & KV315 & AMBP & ITIH4 & TTR \\
\hline Gr1/CG & 0.410 & 4.936 & 0.527 & 0.338 & 0.329 \\
\hline Gr1/Gr2 & 0.565 & 1.243 & 0.530 & 0.428 & 0.332 \\
\hline Gr2/CG & 0.728 & 3.971 & 0.993 & 0.790 & 0.993 \\
\hline$P$-value & 0.01332 & 0.00896 & 0.00023 & -0.471 & 0.00038 \\
\hline
\end{tabular}

Taking into account the results obtained, we can assume that we register a signal from the total fraction of $\kappa$-chains. According to Katzmann, the content of FLC $\lambda$ slightly exceeds the content of FLC $\kappa$ and amounts to $5.7-26.3 \mathrm{mg} / \mathrm{ml}$ for FLC $\lambda$ and 3.3-19.4 $\mathrm{mg} / \mathrm{ml}$ for FLC $\kappa$, with the diagnostic interval of $0.26-1.65$ for the $\kappa / \lambda$ ratio. ${ }^{(7)}$ This indicator is often used as an auxiliary parameter for diagnosing inflammatory reactions, oncohematological diseases, rheumatoid arthritis, and systemic lupus erythematosus in the early stages of development.

There are data on the dynamics of the content of LCs and their ratio in pregnant women in the third trimester, after childbirth and in comparison with non-pregnant women. Lima et al. ${ }^{(6)}$ showed that the median levels of both $\kappa$ and $\lambda$ FLC were significantly higher during the postpartum period than on the day of delivery. The levels of FLC $\kappa$ recovered during the postpartum period were not significantly different from those of the non-pregnant women, whereas the levels of FLC $\lambda$ remained low. Thus, the $\kappa / \lambda$ ratio increased during the postpartum period, which can be related to higher activity of the adaptive immune system. According to Lima et al., ${ }^{(8)}$ this finding is an indicator of B-cell activation occurring after the systemic immunological reaction associated with childbirth. In addition, the increased $\kappa / \lambda$ ratio may indicate polyclonal hypergammaglobulinemia, which occurs during the postpartum period and may be caused by an inflammatory state. Indirectly, polyclonal hypergammaglobulinemia is also indicated by the high level of B-cell-activating factor on the day of delivery and during the postpartum period, rather than during the third trimester of pregnancy.

Jenner et al..$^{(9)}$ argue that the tendency to an increase in circulating $\mathrm{lg}$-LCs is not only an indicator of internal immunological processes, but also a prognostic indicator of the development of preeclampsia and various obstetric complications, including preterm birth.

In conclusion, the results of our study show that KV315 can be considered as the most promising serologic marker for MM in early gestation. It seems appropriate before planning a pregnancy to conduct an additional examination of women who show a high KV315 value in order to predict the outcome of pregnancy. 


\section{Acknowledgments}

This study was supported by the RUDN University Strategic Academic Leadership Program.

\section{Competing Interests} interests.

The authors declare that they have no competing

\section{References}

1. Radzinsky VE, Orazmuradov AA, Savenkova IV, Damirova KF, Haddad H. Preterm labour: an open problem in XXI century. Kuban Scientific Medical Bulletin. 2020;27(4):27-37. [Article in Russian].

2. Non-developing pregnancy. Chapter 14. In: Radzinsky VE, Orazmuradova AA, editors. Early pregnancy. From pregravid preparation to healthy gestation. Moscow: StatusPraesens; 2020:509-537.

3. Svyatova GS, Berezina GM, Salimbaeva DN, Kirikbaeva MS, Murtazalieva AV, Saduakasova KZ. [Genetic aspects of the idiopathic form of recurrent miscarriage. Literature review]. Science\&Healthcare. 2019; 21(4):37-49. [Article in Russian].

4. Aleynik VA, Babich SM, Negmatshaeva XN, Yuldasheva OS. [Features of immunological changes in women with a miscarriage at the presence of autoantibodies]. Molodoi Uchenii. 2017;22(156):411-414. [Article in Russian].

5. Lefranc MP. Immunoglobulin and T Cell Receptor Genes: IMGT( $\left({ }^{\circledR}\right)$ and the Birth and Rise of Immunoinformatics. Front Immunol. 2014 Feb 5;5:22. doi: 10.3389/fimmu.2014.00022. 6. UniProtKB - P01624-KV315_HUMAN) https://www. uniprot.org/uniprot/P01624.

7. Klein C, Schaefer W, Regula JT. The use of CrossMAb technology for the generation of bi- and multispecific antibodies. MAbs. 2016 Aug-Sep;8(6):1010-20. doi: 10.1080/19420862.2016.1197457. Epub 2016 Jun 10. Erratum in: MAbs. 2018 Nov 13;11(1):217.

8. Lima J, Cambridge G, Vilas-Boas A, Martins C, Borrego LM, Leandro M. Serum markers of B-cell activation in pregnancy during late gestation, delivery, and the postpartum period. Am J Reprod Immunol. 2019 Mar;81(3):e13090. doi: 10.1111/aji.13090. Epub 2019 Jan 30.

9. Jenner E. Serum free light chains in clinical laboratory diagnostics. Clin Chim Acta. 2014 Jan 1;427:15-20. doi: 10.1016/j.cca.2013.08.018. 